\title{
VARIACIONES DE $\beta$ CAROTENO EN SANGRE DE VACAS LECHERAS DURANTE EL PERIODO POST PARTO
}

\author{
Eduardo Aguiar-Zalzano ${ }^{1}$ y Augusto Rojas-Bourrillon ${ }^{2}$
}

\section{RESUMEN}

El caroteno es el principal precursor de la vitamina A, la cual es muy importante en los procesos reproductivos e inmunológicos del animal. Durante el estudio se pudo notar que las concentraciones sanguíneas de $\beta$ caroteno caen considerablemente al momento del parto, para posteriormente incrementarse de forma paulatina hasta los 90 días post parto. De igual forma se pudo notar que las vacas que presentaron una concentración sanguínea promedio de $5,87 \mathrm{mg} / \mathrm{ml}(587 \mu \mathrm{g} / \mathrm{dl})$ tuvieron un mayor índice de preñez, si se compara con las vacas que presentaron concentraciones promedios de $4,31 \mathrm{mg} / \mathrm{ml}$ (431 $\mu \mathrm{g} / \mathrm{dl}$ ), sugiriendo una correlación entre las concentraciones de caroteno al parto sobre la instauración temprana de una gestación.

Palabras claves: vitamina A, preñez, periodo de transición, ganado lechero

\section{ABSTRACT}

Blood $\beta$ Carotene variations in dairy cows during the post partum period. The $\beta$ carotene is the main precursor of vitamin $A$, which is very important for the animal in reproductive and immunological processes. Through the study it was noted that levels of $\beta$-carotene in the blood drop considerably at calving and gradually increase again until 90 days post partum. Additionally, it was noted that cows with average blood concentration of $5.87 \mathrm{mg} / \mathrm{ml}(587 \mathrm{mg} / \mathrm{dl})$ had a higher pregnancy rate when compared with cows with a mean concentration of $4.31 \mathrm{mg} / \mathrm{ml}(431 \mathrm{mg} / \mathrm{dl})$, suggesting a correlation between $\beta$ carotene concentrations at calving and early establishment of a pregnancy.

Keywords: Vitamin A, pregnancy, transition period, dairy cattle

\footnotetext{
${ }^{1}$ Universidad de Costa Rica. Maestría Académica en Ciencias Agrícolas y Recursos Naturales con énfasis en Nutrición Animal. Facultad de Ciencias Agroalimentarias. San José, Costa Rica.

zUniversidad de Costa Rica. Centro de Investigaciones en Nutrición Animal, Escuela de Zootecnia. San José, Costa Rica. Autor para correspondencia: augusto.rojas@ucr.ac.cr 


\section{INTRODUCCIÓN}

La vitamina A, conocida como: retinol, axeroftol, biosterol, vitamina antixeroftalmica y vitamina anti infecciosa (Quintela et al., 2008), es esencial en la dieta debido a las funciones vitales que desempeña en el organismo y a la incapacidad del organismo de producirla. El $\beta$ caroteno es el principal precursor de la vitamina A, proviniendo principalmente, en el caso de los rumiantes, de los forrajes (Kaewlamun et al., 2011), siendo almacenado en retinol, luego de ser convertido en el hígado (Bendich y Olson, 1989).

El transporte de los $\beta$ carotenos es por medio del sistema linfático, junto con la grasa, y son almacenados temporalmente en el hígado, para posteriormente ser biotransformado a vitamina A y cumplir un papel importante en la eficiencia reproductiva de las vacas (Kaewlamun et al., 2011).

Debido a que los $\beta$ carotenos no pueden ser sintetizados a partir de otros compuestos, su administración es de gran importancia (Searles y Armstrong, 1870). Sin embargo las concentraciones de $\beta$ caroteno en los alimentos pueden cambiar durante las estaciones climáticas. Por ejemplo, Arikan y Rodway (2001) observaron un descenso de las concentraciones de $300 \mathrm{ppm}$ a $10 \mathrm{ppm}$ en forrajes al finalizar el invierno en países no tropicales.

De igual forma Kalac (2012), demostró que el ensilaje de maíz es una fuente limitada de $\beta$ caroteno. Esta situación también ha sido evaluada en otros forrajes y se ha denominado "síndrome de deficiencia de vitamina A por ensilaje de maíz", en donde se producen pérdidas de 19,59 y $82 \%$ del nivel inicial de $\beta$ carotenos en forrajes verdes, forrajes deshidratados y ensilajes, y henos, respectivamente. De igual forma, otros procesos como la fermentación ruminal también pueden alterar estos compuestos, gran parte de la vitamina $A$ y el $\beta$ caroteno ingerido no es absorbido como tal, pues en solo 12 horas de incubación in vitro, se destruye entre el 60 y $72 \%$ del retinol (Weiss, 1998).

Según Can et al. (1986) y Puis (1994), (citados por Quintela et al. 2008), para animales rumiantes, los niveles normales de vitamina $A$ en sangre deben estar entre 25 y $89 \mu \mathrm{g} / \mathrm{dl}$, mientras que los $\beta$ carotenos se deben encontrar entre 300 y $1.200 \mu \mathrm{g} / \mathrm{dll}$. De acuerdo a lo 
anterior, al encontrar valores inferiores a $7 \mu \mathrm{g} / \mathrm{dl}$ de vitamina $\mathrm{A}$, o su equivalente a 100 $\mu \mathrm{g} / \mathrm{dl}$ de $\beta$ caroteno en sangre, se podría considerar como una deficiencia severa, mientras que para valores intermedios se consideraría como una deficiencia leve. Por lo tanto, la suplementación de $\beta$ caroteno y/o vitamina $A$ en animales con deficiencia $(<300$ $\mu \mathrm{g} / \mathrm{dl}$ y $<25 \mu \mathrm{g} / \mathrm{dl}$, respectivamente) puede tener un efecto beneficioso sobre los parámetros reproductivos de éstos.

Adicionalmente, Arikan y Rodway (2001) observaron, en una zona templada, que cuerpos lúteos colectados entre los meses más calientes (mayo-octubre) contienen una concentración significativamente mayor de $\beta$ caroteno al compararla con las cantidades de éste durante los meses más fríos (noviembre-abril). Esto debido posiblemente a que, durante los meses cálidos, las pasturas y los forrajes verdes proveen una mayor cantidad de $\beta$ caroteno, esto según estudios realizados en países templados. Lo anterior puede ser de gran importancia en la reproducción, ya que el cuerpo lúteo contiene una alta concentración de $\beta$ caroteno y es el encargado de la producción de progesterona (hormona indispensable para la instauración de una gestación) (Quintela et al., 2008). Sin embargo Kaewlamun et al. (2011) no lograron demostrar que la suplementación de $\beta$ caroteno durante el preparto, mejora la actividad ovárica y la producción de progesterona post parto.

Por otro lado, De Ordanza et al. (2009) reportan la necesidad de realizar suplementaciones de $\beta$ caroteno por un largo periodo de tiempo para lograr obtener un impacto positivo en la eficiencia reproductiva. Esos resultados son parecidos a lo observado por Aréchiga et al. (1998), quienes notaron que al suplementar con 400 mg/día de $\beta$ caroteno no se obtiene una mejora reproductiva en las vacas, aunque al suplementar estas mismas vacas por un periodo superior a los 90 días se obtuvo un incremento en la tasa de preñez a los 120 días post parto (35,4\% vs 21,1\%). Estos reportes dejan en evidencia una vez más la necesidad de mejorar el entendimiento de los modelos de $\beta$ carotenos como posibles estimulantes reproductivos e inmunológicos y su acción en el rumen. Esto debido a las posibles interacciones entre componentes de la ración, niveles de suplementación, estrategias alimenticias, momento óptimo de administración y tiempo de suplementación (De Ondarza y Engstrom, 2009).

Al observar las múltiples funciones que tiene la vitamina A en el organismo, y los graves efectos que puede tener su deficiencia en las funciones reproductivas e inmunológicas, se considerar necesario realizar un estudio que evalúe los niveles y variaciones en las Nutrición Animal Tropical 9(2): 91-104. ISSN: 2215-3527/ 2015 
concentraciones de $\beta$ caroteno sanguíneo en vacas lecheras en climas tropicales durante el postparto. Todos los estudios citados anteriormente fueron realizados en zona no tropicales, donde las variaciones climáticas generan cambios significativos en las dietas forrajeras al compararla con países tropicales. El objetivo del presente estudio busca correlacionar la tasa de preñez con los niveles sanguíneos postparto de $\beta$ caroteno en vacas lecheras.

\section{MATERIALES Y MÉTODOS}

El estudio se realizó en la Hacienda la llusión, cantón San Rafael, provincia de Heredia, Costa Rica. La cual se ubica a $1.750 \mathrm{msnm}$, presentando una temperatura media anual de $20{ }^{\circ} \mathrm{C}$ y una precipitación media anual de $2.400 \mathrm{~m}^{3}$. Se recabaron los datos entre los meses de mayo y noviembre (temporada de lluvia).

Se muestrearon 22 vacas Holstein el día del parto y posteriormente cada 30 días por un periodo de 90 días. Durante el desarrollo del ensayo, los animales consumieron una dieta

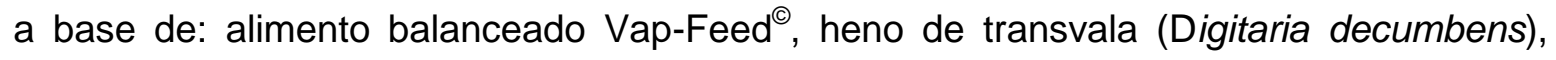
citropulpa peletizada, cáscara de banano, harina de soya y grasa de sobre paso. Los animales se mantuvieron en pastoreo de pasto kikuyo (Kikuyocloa clandestinum)y pasto estrella africana (Cynodon nlemfluensis) de 30 días de edad (Cuadro 1).

A partir del día 60 de lactación, las vacas iniciaron un programa de inseminación artificial, el cual fue realizado por el mismo operario y siguiendo el protocolo a.m. - p.m. La vaca que presentó el celo en la mañana fue inseminada ese mismo día en la tarde y la que presentó el celo en la tarde fue inseminada en la mañana del día siguiente, siendo inseminadas solo aquellas vacas que presentaron un flujo cristalino y abundante. 
Cuadro 1. Aporte de $\beta$ caroteno de los ingredientes de la ración de las vacas durante el periodo de ensayo.

\begin{tabular}{|c|c|c|}
\hline Material & $\begin{array}{c}(\mathrm{Kg} \\
\mathrm{MS} / \mathrm{vaca} / \mathrm{día})\end{array}$ & $\begin{array}{c}\beta \text { Caroteno } \\
(\mathrm{mg} / \mathrm{Kg})\end{array}$ \\
\hline Alimento Balanceado Vap-feed ${ }^{\odot}$ & 6,96 & - \\
\hline Citropulpa peletizada & 0,87 & $0^{\mathrm{a}}$ \\
\hline Cáscara de banano & 2,14 & $36,9^{b}$ \\
\hline Harina de soya & 0,26 & - \\
\hline Grasa de sobrepaso & 0,60 & - \\
\hline Heno de Transvala (Digitaria decumbens) & 1,60 & $0^{\mathrm{a}}$ \\
\hline Pasto Kikuyo (Kikuyocloa clandestinum)* & 5,50 & $222^{a}$ \\
\hline Total & 17,93 & $1.299,96$ \\
\hline
\end{tabular}

${ }^{\mathrm{a}}$ NRC, 1982.

*El dato fue tomado de Ryegrass perenne (Lolium perenne) fresco.

Como se puede observar en el Cuadro 1, las vacas consumieron aproximadamente un total de 1.299,96 mg de $\beta$ caroteno/día, duplicando así los requerimientos sugeridos por Crew (1987) de 300 a 600 mg/día para garantizar la adecuada actividad inmunológica y reproductiva de las vacas.

\section{Muestras de sangre y análisis de $\beta$ caroteno}

Los valores de $\beta$ caroteno en suero se evaluaron en las 22 vacas, por medio de la toma de sangre en la vena coccígea, a los 0, 30, 60 y 90 días post parto (DPP). Se utilizó el sistema de extracción iEx (extracción de $\beta$ caroteno de un solo paso con un disolvente orgánico), seguido de la medición de $\beta$ caroteno utilizando iCheck (BioAnalytGmbH, Teltow, Alemania) el cual realiza la lectura por medio un espectrofotometría portátil (Raila, et al. 2011). 


\section{Análisis estadístico}

Todos los datos fueron analizados mediante el programa Infostat@ y se realizó un análisis de regresión, evaluando el ajuste de la curva mediante el valor de $\mathrm{R}^{2}$. También se realizó un ANOVA para evaluar la influencia de los niveles de $\beta$ caroteno sanguíneo sobre la preñez a los 120 días, comparando las medias a través de un test de Tukey.

\section{RESULTADOS Y DISCUSIÓN}

Al observar los cambios en los niveles de $\beta$ caroteno en el postparto se puede notar que alrededor del parto, los niveles de este son los más bajos durante el periodo estudiado, para posteriormente sufrir un incremento paulatino en los días siguientes. Esta tendencia fue también observada en un estudio realizado por Johnston y Chew (1984), quienes reportaron que el ciclo productivo puede afectar las concentraciones $\beta$ caroteno, reduciendo las concentraciones plasmáticas durante el periodo seco, y alcanzan el punto más bajo cerca del día 4 ó 6 postparto. De igual forma Kaewlamun et al. (2011) demostraron que las concentraciones plasmáticas de $\beta$ caroteno varían durante el ciclo productivo de la vaca, alcanzando el pico más alto un mes después del secado y el nivel inferior 2 semanas después del parto. En contraste, Drackley, (1999) y Baldi, (2005), afirmaron que la mayor necesidad de vitaminas en vacas lecheras, se produce alrededor del parto, cuando estas son expuestas a estrés fisiológico. No obstante, los requerimientos nutricionales para la producción de leche y la secreción de vitaminas con el calostro, no son suficientes para explicar la disminución, por lo que el fraccionamiento de la vitamina también podría ser un factor que afecte los valores normales de vitaminas del animal durante este periodo.

En la presente investigación, debido a las condiciones de la explotación y a que el estudio se realizó durante el periodo de lluvia, se pudo apreciar que ningún animal, durante el muestreo, presentó niveles plasmáticos de $\beta$ caroteno inferiores a 3,0 mg/L (300 $\mu \mathrm{g} / \mathrm{dl})($ Figura 1). Esa es la concentración mínima necesaria reportada por Can et al. (1986) y Puis (1994) (citados por Quintela et al. 2008). 


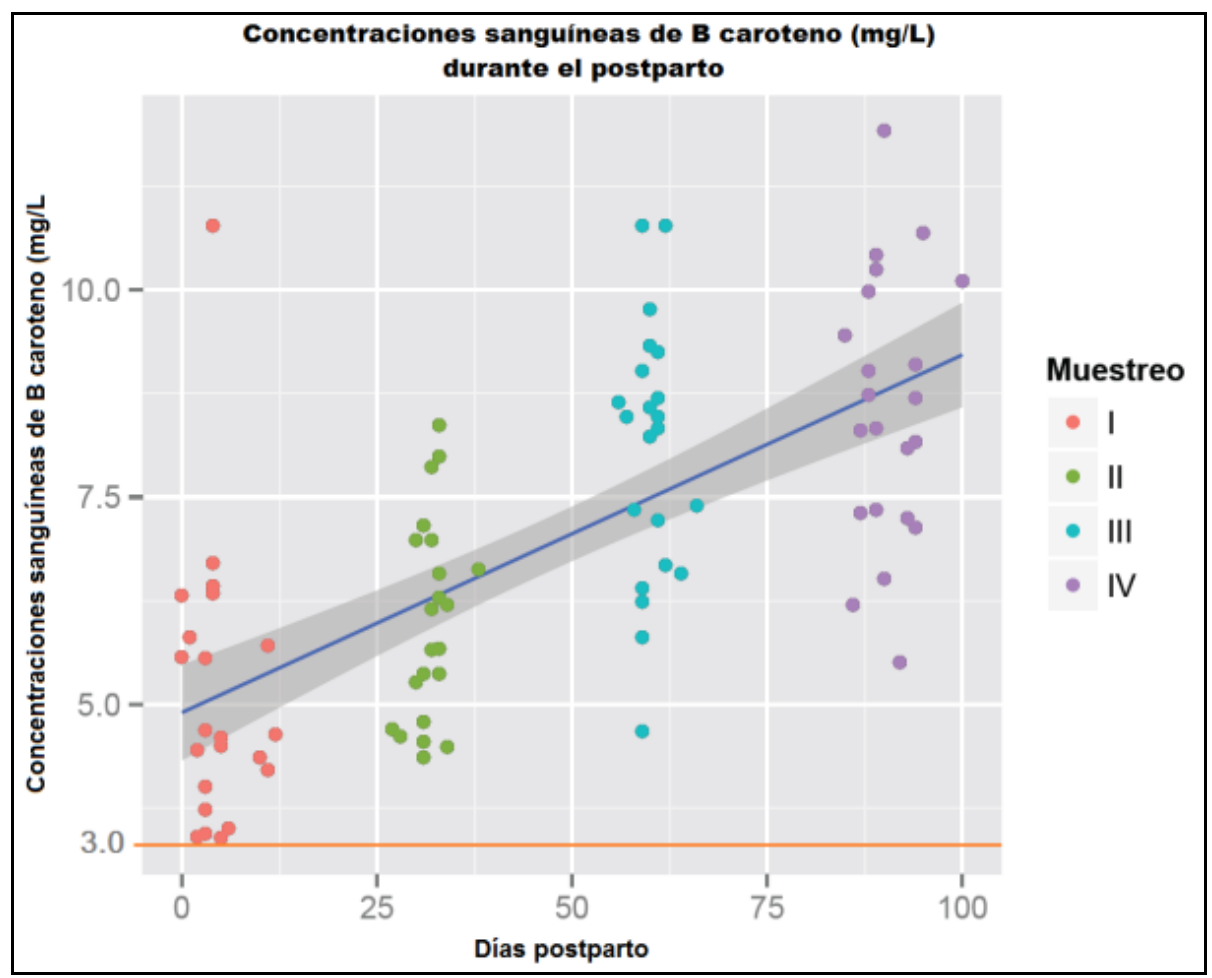

Figura 1. Concentraciones sanguíneas de $\beta$ caroteno $(\mathrm{mg} / \mathrm{L})$ durante el periparto. Línea de regresión: $Y=4,9013+0,0432 X\left(R^{2}=0,4538\right)$

De igual forma se logró obtener una regresión lineal de las concentraciones de $\beta$ caroteno entre los días 0 y 90 postparto. Sin embargo esta demostró una variabilidad considerable entre animales, atribuida posiblemente a los hábitos alimenticios de cada animal en el potrero, alterando así la ingesta diaria de $\beta$ caroteno (Figura 1).

Estos cambios fisiológicos observados en las concentraciones de $\beta$ caroteno en sangre, pueden afectar en gran medida las producciones lecheras orgánicas, como lo demostraron Johansson, et al. (2014), quienes afirman que este tipo de producción es capaz de cubrir los requerimientos de vitaminas $A$ y $E$ de las vacas, sin necesidad de suplementar vitaminas sintéticas. Aunque, alrededor del parto, se hace necesaria la suplementación de éstas, probablemente a un nivel más alto de lo recomendado en la actualidad para explotaciones tradicionales, para evitar así problemas de salud en las vacas y sus terneros. 
Para evaluar la eficiencia reproductiva, se compararon las varianzas de las concentraciones sanguíneas de $\beta$ caroteno en las diversas mediciones $(0,30,60$ y 90 días post parto) con respecto al índice de preñez a los 120 días postparto, notando que durante la primera medición (al parto) el efecto fue significativo. Aquellas vacas que presentaron concentraciones medias de $\beta$ caroteno superiores $(5,87 \mathrm{mg} / \mathrm{L}$ o $587 \mu \mathrm{g} / \mathrm{dl})$ presentaron un mayor índice de preñez a los 120 días, si se compara con aquellas que presentaron una concentración media de $\beta$ caroteno inferior $(4,31 \mathrm{mg} / \mathrm{L}$ o $431 \mu \mathrm{g} / \mathrm{dl})$ $(P<0,05)$ (Cuadro 2). Estos niveles podrían ser garantizados con la adición de $\beta$ caroteno sintético en la época de mayor requerimiento, como lo demostraron Greenberg et al. (1986), observando que las vacas suplementadas con $625 \mathrm{mg}$ de $\beta$ caroteno/día tuvieron niveles plasmáticos superiores a $300 \mu \mathrm{g} / \mathrm{dl}$ en la tercera o cuarta semana post parto, a pesar de su disminución durante el preparto. Por otro lado Wang, et al. (1987) demostraron que la suplementación con $\beta$ caroteno, desde los 30 días preparto hasta los 98 días post parto, no genera ningún beneficio reproductivo en vacas Holstein alimentadas con una dieta comúnmente utilizado en los Estados Unidos. Al comparar los resultados de la presente investigación se podría considerar que la dieta base empleada para la alimentación de estos animales, bajo estas condiciones, cubre los requerimientos de $\beta$ caroteno del animal, por lo que no se haría necesaria una suplementación, aunque Wang et al. (1987) no reportan datos de los aportes de $\beta$ carotenos de las dietas utilizadas en su experimento.

En las vacas evaluadas se obtuvo un índice de preñez a los 120 días de 50\% (11 vacas preñadas), indicando problemas para la instauración temprana de la preñez. Según Goto, et al. (1989), la implantación embrionaria y el reconocimiento materno de la gestación puede ser garantizada con concentraciones plasmáticas de $\beta$ caroteno iguales 0 superiores a $200 \mu \mathrm{g} / \mathrm{dl}$, situación que no coincide con los datos de este muestreo, pues todas las vacas tuvieron concentraciones superiores a ésta durante todo el periodo de estudio.

De igual forma, Pethes, et al. (1985) afirmaron que los bajos niveles de $\beta$ caroteno, son capaces de producir un retraso en la ovulación, formación de cuerpos lúteos y un incremento en la incidencia de quistes; lo que a su vez ocasiona una reducción en la tasas de concepción y abortos temprano. Trojačanec, et al. (2012) obtuvieron resultados similares y notaron que las vacas suplementadas con $500 \mathrm{UI}$ de vitamina A o $200 \mathrm{mg}$ de $\beta$ 
caroteno + $500 \mathrm{UI}$ de vitamina A tuvieron mayores niveles de progesterona al compararlas con las que recibieron solo $\beta$ caroteno o las del grupo control, por lo que las mejoras en los parámetros reproductivos en vacas con problemas crónicos de fertilidad es resultado de la corrección en los aportes de vitamina $A$ o $\beta$ caroteno.

Cuadro 2. Concentraciones sanguíneas de $\beta$ caroteno (mg/L) y su efecto sobre la preñez a los 120 días.

\begin{tabular}{|c|c|c|c|c|}
\hline Muestreo & $\begin{array}{c}\text { Preñez } \\
120 \text { dpp }\end{array}$ & $\begin{array}{c}\text { Concentración } \\
\beta \text { caroteno (mg/L) }\end{array}$ & D.E. & $\begin{array}{c}\text { P valor } \\
\text { (Preñez } 120 \text { dpp) }\end{array}$ \\
\hline I (al parto) & $\begin{array}{l}\mathrm{SI} \\
\mathrm{NO}\end{array}$ & $\begin{array}{l}5,87^{\mathrm{b}} \\
4,31^{\mathrm{a}}\end{array}$ & 0,45 & 0,0237 \\
\hline II (30 dpp) & $\begin{array}{l}\mathrm{SI} \\
\mathrm{NO}\end{array}$ & $\begin{array}{l}6,17 \\
5,83\end{array}$ & 0,37 & 0,5200 \\
\hline III (60 dpp) & $\begin{array}{l}\mathrm{SI} \\
\mathrm{NO}\end{array}$ & $\begin{array}{l}8,62 \\
7,44\end{array}$ & 0,45 & 0,0786 \\
\hline IV (90 dpp) & $\begin{array}{l}\text { SI } \\
\text { NO }\end{array}$ & $\begin{array}{l}9,03 \\
8,10\end{array}$ & 0,47 & 0,1785 \\
\hline
\end{tabular}

Aunque ninguna vaca durante el muestreo presentó concentraciones inferiores a 300 $\mu \mathrm{g} / \mathrm{dl}$, valor mínimo recomendado por Can et al. (1986) y Puis (1994) (citados por Quintela et al. 2008), es importante destacar que esos experimentos fueron realizados en países templados. Sin embargo, según Loven (1988) (citado por Quintela et al. 2008), el estrés térmico puede generar la producción de radicales libres, los cuales son capaces de desencadenar un estrés oxidativo en el animal, causando posibles pérdidas embrionarias. Es por ello que las condiciones tropicales podrían incrementar los requerimientos de $\beta$ caroteno, debido a un estrés oxidativo superior al presentado por las vacas en los países templados. Este incremento en los requerimientos de $\beta$ caroteno en vacas bajo estrés térmico, fue reportado también por Aréchiga et al. (1998) quienes observaron que la suplementación de $\beta$ caroteno, por un periodo de 90 días, en época de estrés térmico, 
logró incrementar la tasa de concepción a los 120 días; mientras que en épocas de confort climático este efecto no se evidenció.

Por otro lado Kaewlamun et al. (2011), demostró que la suplementación de $1 \mathrm{~g}$ de $\beta$ caroteno/vaca/día es capaz de generar efectos positivos sobre la actividad ovárica y sobre la recuperación uterina, pues fue capaz de reducir las concentraciones de hidroxiprolina sangre y de aumentar los niveles de leucocitos en útero y cuello uterino, demostrando también un efecto sobre el sistema inmunológico de la vaca. Este factor se debe considerar en la implantación embrionaria, pues una reactivación ovárica y una recuperación uterina pronta acortarían el tiempo parto-gestación, incrementando así la eficiencia de la productiva de la explotación.

\section{CONSIDERACIONES FINALES}

El $\beta$ caroteno tiene un efecto ya reportado, sobre la reproducción y el control de los factores anti oxidantes del organismo, por ello es necesario garantizar los valores adecuados en el postparto, momento en el que los valores de este compuesto se ven reducidos y se requiere una clara recuperación de estos para alcanzar una preñez en los tiempos estipulados y lograr así una producción eficiente.

Los niveles de $\beta$ caroteno tiene el valor más bajo al momento del parto y sus valores van incrementándose de manera paulatina en los siguientes 3 meses postparto. Sin embargo y debido al alto aporte de forraje en la explotación evaluada, los valores de $\beta$ caroteno sanguíneo nunca fueron iguales o inferiores a $3 \mathrm{mg} / \mathrm{ml}$ o $300 \mu \mathrm{g} / \mathrm{dl}$. Sin embargo, vacas con valores superiores a 5,87 mg/ml (587 $\mu \mathrm{g} / \mathrm{dl})$ tuvieron un mayor índice de preñez, si se compara con las vacas que presentaron concentraciones promedios de 4,31 mg/ml (431 $\mu \mathrm{g} / \mathrm{dl}$ ), pudiendo indicar una diferencia en los requerimientos de $\beta$ caroteno de vacas en el trópico. Por ello sería recomendable realizar muestreos en diferentes zonas y con un mayor número de animales, con el fin de generar más datos y una concentración mínima de $\beta$ caroteno en sangre adaptado a las condiciones del país. 


\section{AGRADECIMIENTO}

Un especial agradecimiento al propietario y a los empleados de la Hacienda La llusión, por facilitarnos los animales y las instalaciones para desarrollar este estudio y a la empresa DSM Nutritional Products por su colaboración en el préstamo del equipo utilizado en las mediciones empleadas.

\section{LITERATURA CONSULTADA}

Aréchiga, C., Staples, C., McDowell L., H Ansen, P. 1998. Effects of timed insemination and supplemental $\beta$-Carotene on reproduction and milk yield of dairy cows under heat stress. Journal of Dairy Science 81(2): 390-402.

Arikan, Ş., Rodway, R. 2001. Seasonal variation in bovine luteal concentrations of $\beta$ carotene. Turk Journal Veterinary Animals Science. 25: 165-168

Baldi, A. 2005. Vitamin E in dairy cows. Livestock Production Science. 98:117-122.

Bendich, A., Olson, J. 1989. Biological actions of carotenoids. FASEB Journal 3: 19271932.

Chew, B. 1987. Vitamin A and $\beta$-carotene in host defense. Journal of Dairy Science. 70: 2732- 2743.

De Ondarza, M., Engstrom, M. 2009. Can beta-carotene help dairy reproduction?. Feedstuffs 81(40): 1-5

De Ordanza, M., Wilson, J., Engstrom, M. 2009. Case study: effect of supplemental $\beta$ carotene on yield of milk and milk component and reproduction of dairy cow. The Professional Animal Scientist 25: 510-516.

Drackley, J. K. 1999. Biology of dairy cows during the transition period: The final frontier? Journal of Dairy Science. 82: 2259-2273. 
Goto, K., Kajisa, O., Ezoe, K., Nakanishi, Y., Ogawa, K., Tasaki, M., Ohta, H., Inohae, S., Tateyama, S., Kawabata, T. 1989. Relationship between plasma beta carotene concentration and embryo quality in superovulated Japanese black cattle. Memoirs of the Faculty of Agriculture Kagoshima University. 25:113-117.

Greenberg, L., Bristol, F., Murphy, B., Laarveld, B. 1986. Beta-carotene does not influence fertility in beef heifers. Theriogenology. 26(4):491-508.

Johansson, B., Persson, K., Jensen, S., Lindqvist, H., Nadeau, E. 2014. Status of vitamins $E$ and $\beta$ carotene and heath in organic dairy cows fed a diet without synthetic vitamins. Journal of Dairy Science. 97(3): 1682-1692.

Johnston, L., Chew, B. 1984. Peripartumchanges of plasma and milk vitamin A and beta-carotene among dairy cows with or without mastitis Journal of Dairy Science. 67: 1832-1840.

Kaewlamun, W., Okouyi, M., Humblot, P., Techakumphu, M., Ponter, A. 2011. Does supplementing dairy cows with $\beta$-carotene during the dry period affect postpartum ovarian activity, progesterone, and cervical and uterine involution?. Theriogenology 75: 1029-1038.

Kalač, P. 2012. Carotenoids, ergosterol and tocopherols in fresh and preserved herbage and their transfer to bovine milk fat and adipose tissues: A review. Journal of Agrobiology 29(1): 1-13.

Monge-Rojas, R., Campos H. 2013. Tabla de composición de alimentos de Costa Rica: Carotenoides y Tocoferoles. INCIENSA. Tres Ríos, Costa Rica.

NationalResearch Council. 1982. UnitedStates-Canadian tables of feedcomposition. Tercera edición. NationalAcademycPress, Washington DC.

Pethes, B. G., E. Horvath, M. Kulcsar, G. Huszenicza, G. Somorjai, B. Varga, J. Harasztil. 1985. In vitro progesterone production of corpus luteum cells of cows fed low and high levels of beta-carotene. Zbl. Vet. Med. A. 32: 289-296.

Quintela, L., Diaz, C., Becerra, J., Alonso, G., Gracia, S., Herradon, P. 2008. Papel del $\beta$ caroteno y la vitamina $A$ en la reproducción en el ganado vacuno: revisión. ITEA 104 (3): 399-410.

Raila, J., Enjalbert F., Mothes, R., Hurtienne, A., Schweigert, F. 2011. Determination of ßcarotene in whole blood of cattle: Comparison of a new cow-side assay with HPLC. Veterinary Clinical Pathology. Brief Communication. 
Sales, J., Dias, L., Viveiros, A., Pereira, M., Souza, J. 2008. Embryo production and quality of Holstein heifers and cows supplemented with $\beta$-carotene and tocopherol. Animal Reproduction Science 106: 77-89

Searles, S., Armstrong, J. 1970. Vitamin E, vitamin A, and carotene contents of alberta butter. Journal of Dairy Science. 53: 150-154.

Trojačanec, S., Boboš, S., Pajić, M. 2012. Influence of B carotene and vitamin A supplementation on the ovarian activity of dairy cows with chronic fertility impairment. VeterinarskiArchiv 82 (6): 567-575.

Wang, J., Hafi, C., Owen, F., Larson L. 1987. Effect of beta-carotene supplementation on periparturient health and reproduction of Holstein cows. Animal Reproduction Science. 15:139-144

Weiss, W. 1998. Requirements of fat-soluble vitamins for dairy cow. Journal of Dairy Science. 81: 2493-2501. 